\title{
Modified gravity from the quantum part of the metric
}

\author{
Vladimir Dzhunushaliev ${ }^{1,2,3,4, a}$, Vladimir Folomeev ${ }^{2,3, b}$, Burkhard Kleihaus $^{4, c}$, Jutta Kunz ${ }^{4, d}$ \\ ${ }^{1}$ Department of Theoretical and Nuclear Physics, KazNU, Almaty 050040, Kazakhstan \\ 2 IETP, Al-Farabi Kazakh National University, Almaty 050040, Kazakhstan \\ ${ }^{3}$ Institute of Physicotechnical Problems and Material Science of the NAS of the Kyrgyz Republic, \\ 265 a, Chui Street, Bishkek 720071, Kyrgyz Republic \\ ${ }^{4}$ Institut für Physik, Universität Oldenburg, Postfach 2503, 26111 Oldenburg, Germany
}

Received: 11 December 2013 / Accepted: 14 January 2014 / Published online: 30 January 2014

(C) The Author(s) 2014. This article is published with open access at Springerlink.com

\begin{abstract}
It is shown that if a metric in quantum gravity can be decomposed as a sum of classical and quantum parts, then Einstein quantum gravity looks approximately like modified gravity with a nonminimal interaction between gravity and matter.
\end{abstract}

\section{Introduction}

We know two strongly nonlinear physical theories: quantum chromodynamics and gravity. The nonlinearities in the two theories are different: in quantum chromodynamics, the nonlinearity is connected with potential terms but in gravity the nonlinearity appears in kinetic terms. The quantization of both theories has severe problems, because we only have quantization techniques for weakly interacting fields. In the 1950s, Heisenberg [1] has investigated a nonlinear spinor field theory and worked out a nonperturbative technique for such a kind of quantization. The essence of his method is to write an infinite set of equations for all Green's functions. Such a set of equations probably cannot be solved analytically and a way to solve such a set is to cut it off to obtain a finite set, taking into account some physical arguments for the cutoff. This situation is similar to turbulence modeling, where there exists a similar set of equations for all cumulants (see Ref. [2] for details).

The idea presented here is as follows: we consider a physical system in quantum gravity where the quantum metric $\mathcal{G}$ can be represented as a sum of a classical part, $g$, and a quantum part $\widehat{g}$. In doing so, we here assume that the expectation value of the quantum part, $\langle\widehat{g}\rangle$, is not equal to zero. This gives

\footnotetext{
a e-mail: v.dzhunushaliev@gmail.com

be-mail: vfolomeev@mail.ru

c e-mail: b.kleihaus@uni-oldenburg.de

de-mail: jutta.kunz@uni-oldenburg.de
}

rise to a modification of Einstein gravity that results in the appearance of a nonminimal interaction between gravity and matter. To calculate the modified gravitational Lagrangian, we also assume that the expectation value of the quantum part of the metric can be approximately presented as an expression which depends on the classical part of the metric. Taking into account the aforementioned decomposition of the metric and retaining only terms up to first order in $\langle\widehat{g}\rangle$, we find the corresponding matter Lagrangian. As a result, we obtain modified gravity with a nonminimal interaction between gravity and matter.

Modified gravity theories are used both in modeling the evolution of the early Universe and in describing its present accelerated expansion.

In particular, in recent years much success has been achieved in models where dark energy is described on the basis of modified gravity (for a review, see Refs. [3,4]).

\section{Nonperturbative quantization technique}

When quantizing gravity, there are several stages for performing this procedure. In the first step, the metric is considered as an ordinary (tensor) field. In the next step, one considers changes of topology, metric signature, etc. Here we consider only the first stage of the quantizing procedure, using Heisenberg's nonperturbative technique. According to this technique, one has to use either the Einstein equations for operators of the metric and connections or (equivalently) an infinite set of equations for all Green's functions.

The operator Einstein equations are

$$
\hat{R}_{\mu \nu}-\frac{1}{2} \hat{g}_{\mu \nu} \hat{R}=\varkappa \hat{T}_{\mu \nu}
$$


where all geometrical quantities are defined in the usual manner from the corresponding operators

$\hat{R}_{\mu \nu}=\hat{R}_{\mu \rho \nu}^{\rho}$,

$\hat{R}_{\sigma \mu \nu}^{\rho}=\frac{\partial \hat{\Gamma}_{\sigma \nu}^{\rho}}{\partial x^{\mu}}-\frac{\partial \hat{\Gamma}_{\sigma \mu}^{\rho}}{\partial x^{\nu}}+\hat{\Gamma}_{\tau \mu}^{\rho} \hat{\Gamma}_{\sigma \nu}^{\tau}-\hat{\Gamma}_{\tau \nu}^{\rho} \hat{\Gamma}_{\sigma \mu}^{\tau}$.

$\hat{\Gamma}_{\mu \nu}^{\rho}=\frac{1}{2} \hat{g}^{\rho \sigma}\left(\frac{\partial \hat{g}_{\mu \sigma}}{\partial x^{\nu}}+\frac{\partial \hat{g}_{\nu \sigma}}{\partial x^{\mu}}-\frac{\partial \hat{g}_{\mu \nu}}{\partial x^{\sigma}}\right)$.

Heisenberg's technique offers to use an infinite set of equations for all Green's functions, which can be written as follows:

$\langle Q| \hat{g}\left(x_{1}\right) \cdot($ Eq. 1) $|Q\rangle=0$,

$\langle Q| \hat{g}\left(x_{1}\right) \hat{g}\left(x_{2}\right) \cdot($ Eq. 1$)|Q\rangle=0$,

$\cdots=0$,

$\langle Q|$ the product of $g$ at different points $\left(x_{1}, \ldots, x_{n}\right)$

$$
\text { - (Eq.1) }|Q\rangle=0 \text {, }
$$

where $|Q\rangle$ is a quantum state (see Ref. [5] for details).

In all likelihood the set of Eqs. (5)-(8) cannot be solved analytically. But there are two possibilities to find an approximate solution to these equations. The first way consists in cutting off the set of equations by using some decomposition of the form $G_{m+n} \approx G_{m} G_{n}$ (here $G_{i}$ is an $i$-point Green's function) and taking into account only the first $p<m+n$ equations. The second way is to take some functional (for example, an action) and to average it using some assumptions about expectation values of metric operators.

Here we use the second way, decomposing the metric operator $\hat{g}_{\mu \nu}$ into classical and quantum parts and considering the expectation value of the quantum part as being non-zero. We then calculate the expectation value of the Lagrangian to first order in $\langle\delta g\rangle$. These calculations are performed in the manner similar to the ones used in Ref. [6] when considering quantum torsion.

\section{Decomposition of the quantum metric}

The key idea we are dealing with here is that the quantum metric $\mathcal{G}_{\mu \nu}$ can be decomposed into two parts: the classical metric $g_{\mu \nu}$ and the quantum metric $\widehat{g}_{\mu \nu}$

$\mathcal{G}_{\mu \nu}=g_{\mu \nu}+\widehat{g}_{\mu \nu}$

together with the assumption that

$\left\langle\widehat{g}_{\mu \nu}\right\rangle \neq 0$.

In order to derive the modification of the Lagrangian induced by the quantum corrections $\left\langle\widehat{g}_{\mu \nu}\right\rangle$, we have to expand the Lagrangian to first order in $\left\langle\widehat{g}_{\mu \nu}\right\rangle$. To do this, we start from the Einstein-Hilbert Lagrangian

$\mathcal{L}_{\mathcal{G}}=-\frac{c^{2}}{2 \varkappa} \sqrt{-\mathcal{G}} R$

where $\varkappa=8 \pi G / c^{2}$. Next, to find corrections to this Lagrangian induced by the quantum corrections $\left\langle\widehat{g}_{\mu \nu}\right\rangle$, we assume that the Lagrangian can be expanded in the following manner:

$\mathcal{L}_{\mathcal{G}}(g+\widehat{g}) \approx \mathcal{L}_{g}(g)+\frac{\delta \mathcal{L}_{g}}{\delta g^{\mu \nu}} \widehat{g}^{\mu \nu}=\mathcal{L}_{g}(g)+\sqrt{-g} G_{\mu \nu} \widehat{g}^{\mu \nu}$,

where $G_{\mu \nu}$ is the Einstein tensor, and

$\mathcal{L}_{g}(g)=-\frac{c^{2}}{2 \varkappa} \sqrt{-g} R$

denotes the classical (nonquantum) Lagrangian. In turn, the expectation value of $\frac{\delta \mathcal{L}}{\delta g^{\mu \nu}} \widehat{g}^{\mu \nu}$ can be represented as follows:

$\left\langle\frac{\delta \mathcal{L}_{g}}{\delta g^{\mu \nu}} \widehat{g}^{\mu \nu}\right\rangle=\frac{\delta \mathcal{L}_{g}}{\delta g^{\mu \nu}}\left\langle\widehat{g}^{\mu \nu}\right\rangle=\sqrt{-g} G_{\mu \nu}\left\langle\widehat{g}^{\mu \nu}\right\rangle$

Now we have to make some physically reasonable assumptions about the expectation value $\left\langle\widehat{g}^{\mu v}\right\rangle$. We assume the following.

- The expectation value $\left\langle\widehat{g}^{\mu v}\right\rangle$ is non-zero:

$\left\langle\widehat{g}_{\mu \nu}\right\rangle \neq 0$.

- As a starting approximation, $\left\langle\widehat{g}_{\mu \nu}\right\rangle$ can be expressed in terms of some tensor of rank two constructed from the metric $g_{\mu \nu}$ :

$\left\langle\widehat{g}_{\mu \nu}\right\rangle=K_{\mu \nu}$.

- The tensor $K_{\mu \nu}$ should have the same symmetry as the metric $g_{\mu \nu}$ :

$K_{\mu \nu}=K_{\nu \mu}$

- Taking into account the symmetry properties mentioned above, one can see that there exist the following possibilities of choosing the tensor $K_{\mu \nu}$ :

- $K_{\mu \nu}$ is proportional to the metric tensor:

$$
K_{\mu \nu} \propto g_{\mu \nu} .
$$

- $K_{\mu \nu}$ is proportional to the Ricci tensor:

$$
K_{\mu \nu} \propto \frac{R_{\mu \nu}}{R} .
$$


- The proportionality coefficient in the expressions (17) and (18) should be some invariant. Consequently, it must have the form $F\left(R, R_{\mu \nu} R^{\mu \nu}, \ldots\right)$. This yields

$$
\left\langle\widehat{g}_{\mu \nu}\right\rangle=K_{\mu \nu}=F\left(R, R_{\mu \nu} R^{\mu \nu}, \ldots\right) g_{\mu \nu} ;
$$

$$
\left\langle\widehat{g}_{\mu \nu}\right\rangle=K_{\mu \nu}=F\left(R, R_{\mu \nu} R^{\mu \nu}, \ldots\right) \frac{R_{\mu \nu}}{R}
$$

- or a linear combination of (19) and (20). For example, it could be the Einstein or Schouten tensor.

The coefficient $F$ should be very small as $g_{\mu \nu} \rightarrow \eta_{\mu \nu}$, where $\eta_{\mu \nu}$ is the Minkowski metric.

Thus the Lagrangian (12) with the aforementioned assumptions about the expectation value of the quantum part $\widehat{g}_{\mu \nu}$ of the metric $\mathcal{G}_{\mu \nu}$ has the form

$\left\langle\mathcal{L}_{\mathcal{G}}(g+\widehat{g})\right\rangle \approx-\frac{c^{2}}{2 \varkappa} \sqrt{-g}\left(R+G_{\mu \nu} K^{\mu \nu}\right)$.

Hence we see that the quantum corrections coming from a non-zero expectation value of the nonperturbatively quantized metric give rise to modified gravity theories.

Let us now perform similar calculations for the matter Lagrangian, applying the decomposition (9). For simplicity, we consider a scalar field for which the Lagrange density is

$\mathcal{L}_{m}^{\mathcal{G}}=\sqrt{-\mathcal{G}}\left[\frac{1}{2} \nabla^{\mu} \phi \nabla_{\mu} \phi-V(\phi)\right] \equiv \sqrt{-\mathcal{G}} \mathcal{L}_{m}(g)$,

with the classical Lagrangian

$\mathcal{L}_{m}(g)=\frac{1}{2} \nabla^{\mu} \phi \nabla_{\mu} \phi-V(\phi)$.

To begin with, we expand the quantum Lagrange density $\mathcal{L}_{m}^{\mathcal{G}}$ from Eq. (22) as follows:

$\mathcal{L}_{m}^{\mathcal{G}}(g+\widehat{g}) \approx \sqrt{-g} \mathcal{L}_{m}(g)+\frac{\delta \sqrt{-g} \mathcal{L}_{m}}{\delta g^{\mu \nu}} \widehat{g}^{\mu \nu}$

The first variation of $L_{m}$ is well known:

$\frac{\delta \sqrt{-g} \mathcal{L}_{m}}{\delta g^{\mu \nu}}=\frac{\sqrt{-g}}{2}\left[\nabla_{\mu} \phi \nabla_{\nu} \phi-g_{\mu \nu} \mathcal{L}_{m}\right]=\frac{\sqrt{-g}}{2} T_{\mu \nu}$,

where $T_{\mu \nu}$ is the energy-momentum tensor. Consequently, we have

$\left\langle\mathcal{L}_{m}^{\mathcal{G}}(g+\widehat{g})\right\rangle=\sqrt{-g}\left[\frac{1}{2} \nabla^{\mu} \phi \nabla_{\mu} \phi-V(\phi)+\frac{1}{2} T_{\mu \nu} K^{\mu \nu}\right]$.
Thus we have obtained a scalar field nonminimally coupled to gravity.

Let us now consider a few cases with various tensors $K_{\mu \nu}$.

3.1 The case $K_{\mu \nu}=g_{\mu \nu} \alpha$

Substituting (19) into (21) and (25), we obtain

$$
\begin{aligned}
\left\langle\mathcal{L}_{\mathcal{G}}(g+\widehat{g})\right\rangle & \approx-\frac{c^{2}}{2 \varkappa}(1-\alpha) R \sqrt{-g}, \\
\left\langle\mathcal{L}_{m}^{\mathcal{G}}(g+\widehat{g})\right\rangle & \approx \sqrt{-g}\left[\mathcal{L}_{m}+\alpha T_{\mu}^{\mu}\right] .
\end{aligned}
$$

It is seen that Eq. (26) assumes the redefinition of the gravitational constant $\varkappa$.

\subsection{The case $K_{\mu v}=-\Lambda \frac{g_{\mu \nu}}{R}$}

Again, substituting (19) in (21) and (25), we obtain

$$
\begin{aligned}
\left\langle\mathcal{L}_{\mathcal{G}}(g+\widehat{g})\right\rangle & \approx-\frac{c^{2}}{2 \varkappa}(R+\Lambda) \sqrt{-g}, \\
\left\langle\mathcal{L}_{m}^{\mathcal{G}}(g+\widehat{g})\right\rangle & \approx \sqrt{-g}\left[\mathcal{L}_{m}-\frac{\Lambda}{R} T_{\mu}^{\mu}\right] .
\end{aligned}
$$

Hence we see from (28) that we have obtained Einstein gravity with a cosmological $\Lambda$-term and matter nonminimally coupled to gravity [see Eq. (29)]. If $\Lambda / R \ll 1$ then the coupling between matter and gravity becomes minimal, and we have

$$
\begin{aligned}
& \langle\mathcal{L}(g+\widehat{g})\rangle=\left\langle\mathcal{L}_{\mathcal{G}}(g+\widehat{g})+\mathcal{L}_{m}^{\mathcal{G}}(g+\widehat{g})\right\rangle \\
& \approx \sqrt{-g}\left[-\frac{c^{2}}{2 \varkappa}(R+\Lambda)+\frac{1}{2} \nabla^{\mu} \phi \nabla_{\mu} \phi-V(\phi)\right] .
\end{aligned}
$$

3.3 The case $K_{\mu \nu}=\alpha R_{\mu \nu}$

Substituting (20) into (21) and (25), we obtain

$$
\begin{aligned}
\left\langle\mathcal{L}_{\mathcal{G}}(g+\widehat{g})\right\rangle & \approx-\frac{c^{2}}{2 \varkappa}\left(R-\frac{\alpha}{2} R^{2}+\alpha R_{\mu \nu} R^{\mu \nu}\right) \sqrt{-g} \\
\left\langle\mathcal{L}_{m}^{\mathcal{G}}(g+\widehat{g})\right\rangle & \approx \sqrt{-g}\left(\mathcal{L}_{m}+\frac{\alpha}{2} T_{\mu \nu} R^{\mu \nu}\right)
\end{aligned}
$$

where the dimensions of $\alpha$ is $\mathrm{cm}^{2}$.

$$
3.4 \text { The case } K_{\mu \nu}=\alpha G_{\mu \nu}
$$

Representing the linear combination of (19) and (20) as the Einstein tensor and substituting it into (21) and (25), we obtain

$$
\begin{aligned}
\left\langle\mathcal{L}_{\mathcal{G}}(g+\widehat{g})\right\rangle & \approx-\frac{c^{2}}{2 \varkappa}\left(R+\alpha G_{\mu \nu} G^{\mu \nu}\right) \sqrt{-g} \\
\left\langle\mathcal{L}_{m}^{\mathcal{G}}(g+\widehat{g})\right\rangle & \approx \sqrt{-g}\left(\mathcal{L}_{m}+\frac{\alpha}{2} G^{\mu \nu} T_{\mu \nu}\right) .
\end{aligned}
$$




\section{Discussion and conclusions}

Thus we have shown that if a metric in quantum gravity can be represented as a sum of classical and quantum parts then such a gravitating physical system looks approximately as being described by modified gravity, in which a nonminimal interaction between matter and gravity is present. The modification of gravity occurs as a consequence of the presence of a non-zero expectation value of the quantum part of the metric.

In particular, proceeding along these lines, by a specific choice for the expectation value of the quantum metric, we can obtain a $\Lambda$-term as a consequence of the fluctuating part of the metric. Such a model then explains qualitatively the smallness of the cosmological term: it is just the expectation value of quantum fluctuations of the metric. At the present epoch these fluctuations should be very small. Following this way, we have also shown that quantum fluctuations lead to a nonminimal interaction between gravity and matter.

We note here that attempts to obtain the cosmological constant from quantum effects are not new. In curved spacetime the regularization of quantum fields gives rise to a one-loop effective Lagrangian for the gravitational field, which (for example, for an FRW universe) becomes $\mathcal{L}_{\text {eff }}=$ $\Lambda_{\infty}+R / 16 \pi G_{\infty}+\alpha_{\infty} R^{2}+\beta_{\infty} R_{\mu \nu} R^{\mu \nu}$ (for details, see e.g. Refs. [7-9]). Unfortunately, such calculations do not give good values for the induced cosmological constant. For example, for the best QCD estimation the vacuum energy density is $\rho_{Q C D} \sim 10^{-3} \mathrm{GeV}^{4}$, which is considerably larger than the observed value $\rho_{v a c} \sim 10^{-47} \mathrm{GeV}^{4}$. In contrast, in our approach employed here quantum corrections arise from the nonperturbative quantization of the metric, and not from the perturbative quantization of various types of fields (scalar, electromagnetic, QCD).

It must be emphasized that the model under consideration assumes that for each quantum state $|Q\rangle$ there exists a unique function $F\left(R, R_{\mu \nu} R^{\mu \nu}, \ldots\right)$. Thus, one can say that $F$-gravities are dynamical ones in the sense that for different quantum states we have different functions $F$.
Acknowledgments This work was supported by the Volkswagen Stiftung. V.D. and V.F. acknowledge support from a grant in fundamental research in natural sciences by the Ministry of Education and Science of Kazakhstan. B. K. and J. K. acknowledge support by the DFG Research Training Group 1620 "Models of Gravity".

Open Access This article is distributed under the terms of the Creative Commons Attribution License which permits any use, distribution, and reproduction in any medium, provided the original author(s) and the source are credited.

Funded by SCOAP ${ }^{3}$ / License Version CC BY 4.0.

\section{References}

1. W. Heisenberg, Introduction to the unified field theory of elementary particles, Max - Planck - Institut für Physik und Astrophysik. (Interscience Publishers, London, New York, Sydney, 1966)

2. D.C. Wilcox, Turbulence Modeling for CFD (DCW Industries, Inc., La Canada, California, 1994)

3. S.'i. Nojiri, S.D. Odintsov, Phys. Rept. 505, 59 (2011). [arXiv:1011. 0544 [gr-qc]]

4. K. Bamba, S. Capozziello, S.'i. Nojiri, S.D. Odintsov. Astrophys. Space Sci. 342, 155 (2012). [arXiv:1205.3421 [gr-qc]]

5. V. Dzhunushaliev, Int. J. Mod. Phys. D 21, 1250042 (2012). [arXiv: 1201.1069 [gr-qc]]

6. V. Dzhunushaliev, V. Folomeev, B. Kleihaus, J. Kunz, Phys. Lett. B 719, 5 (2013). [arXiv: 1210.5878 [gr-qc]]

7. V. Sahni, A.A. Starobinsky, Int. J. Mod. Phys. D 9, 373 (2000). [arXiv:astro-ph/9904398]

8. N.D. Birrell, P.C.W. Davies, Quantum Fields in Curved Space (Cambridge University Press, Cambridge, 1982)

9. A.A. Grib, S.G. Mamaev, V.M. Mostepanenko, Vacuum Quantum Effects in Strong Fields (Friedmann Laboratory Publishing, St. Petersburg, 1994) 